Original Paper http://ajol.info/index.php/ijbcs http://indexmedicus.afro.who.int

\title{
Facteurs de risque liés à l'apparition de la maladie de Gumboro en aviculture semi-intensive au Sénégal
}

\author{
Alkaly BADJI ${ }^{1 *}$, Modou MOUSTAPHA LO ${ }^{2}$ et Rianatou Bada ALAMBEDJI ${ }^{3}$ \\ ${ }^{1}$ Université de Thiès, Institut Supérieur de Formation Agricole et Rurale, BP 54 Bambey, Sénégal. \\ ${ }^{2}$ ISRA/LNERV, Laboratoire National de l'Elevage et de Recherches Vétérinaires, \\ BP 2057 Dakar-Hann, Sénégal. \\ ${ }^{3}$ EISMV, Ecole Inter-Etats des Sciences et Médecine Vétérinaires, BP 5027 Dakar-Fann, Sénégal. \\ *Auteur correspondant ; E-mail : badjialkaly@yahoo.fr; Tel : +221 775665609
}

\section{RESUME}

Au Sénégal, la maladie de Gumboro constitue un véritable obstacle pour le développement de l'aviculture semi-intensive. Une étude épidémiologique portant sur cette affection dans les exploitations semiintensives a été conduite en décembre 2015 dans les régions de Dakar et Thiès. Elle a consisté à évaluer l'association entre des facteurs de risque et l'apparition de la maladie. Pour cela, les gestionnaires de 56 exploitations infectées et 112 indemnes ont constitué les cibles de cette enquête. Les foyers étaient suspectés sur la base des manifestations cliniques et confirmés par la détection du virus avec la RT-PCR. Ainsi, les résultats indiquent une faible liaison entre la présence d'oiseaux domestiques ou sauvages et l'apparition de la maladie (Odds Ratio $=1,44)$. Par contre, la présence de rongeurs (Odds $=22$ ), la gestion des cadavres (Odds Ratio $=7,56)$ et l'accès des personnes étrangères (Odds Ratio $=4,47)$ sont fortement liés à l'apparition de la maladie. La sensibilisation sur la gestion des poulets infectés et morts contribuerait à limiter la dissémination $\mathrm{du}$ virus et les pertes attribuables à la maladie.

(C) 2017 International Formulae Group. All rights reserved.

Mots clés : Epidémiologie, facteurs de risque, Odds Ratio, Sénégal, Maladie de Gumboro.

\section{Risk factors related to the appearance of Gumboro disease in semi-intensive poultry farms in Senegal}

\section{ABSTRACT}

In Senegal, Gumboro disease is a real obstacle to the development of semi-intensive poultry farming. An epidemiological study of this disease in semi-intensive poultry farms was carried out in December 2015 in the regions of Dakar and Thiès. The study consisted to assess the association between risk factors at the onset of the disease. To do so, 56 chicken coop managers of infected farms and 112 disease-free farms were targeted for the survey. The outbreaks of infectious bursal disease virus were suspected on the basis of the clinical manifestations, and confirmed by the detection of virus with RT-PCR. Thus, the results indicated that a weak linkage between the presence of domestic or wild birds and the onset of the disease (Odds Ratio $=1.44$ ). On the other hand, the presence of rodents (Odds $=22$ ), cadaver management (Odds Ratio $=7.56$ ) and the access of 
foreign people in the farm (Odds Ratio $=4.47$ ) are strongly related to the onset of Gumboro disease. Sensitization on the management of infected and dead chickens would help to limit the spread of the virus and the losses attributable to the disease.

(C) 2017 International Formulae Group. All rights reserved.

Keywords: Epidemiology, Risk factor, Odds ratio, Senegal, Gumboro, disease.

\section{INTRODUCTION}

La maladie de Gumboro est l'une des affections qui menace perpétuellement l'industrie aviaire dans le monde (Muller et al., 2003) depuis son apparition, en 1950, dans la ville de Gumboro de l'Etat de Delaware aux Etats Unis d'Amérique. Elle est due à un virus appartenant à la famille des Birnaviridae, au genre Avibirnavirus. Le virus infecte les lymphocytes B de la bourse de Fabricius (BF) et entraine une immunodépression des jeunes poulets de 3 à 6 semaines d'âge (Sharma et al., 2000).

En Afrique, particulièrement dans la sous-région Ouest, des foyers de la maladie ont été observés dans plusieurs pays depuis plusieurs décennies. Au Sénégal, elle est diagnostiquée pour la première fois en 1975 dans des élevages avicoles semi-intensifs de la région de Dakar (Rabson, 2010 ; Badji et al., 2017). Depuis, elle constitue un véritable obstacle au développement de l'aviculture sénégalaise par les mortalités et les pertes de croissance qu'elle provoque. L'environnement joue un grand rôle dans la dissémination du virus de la maladie. Le virus est excrété dans les fientes et se propage facilement par contact direct, par voie orale, via ces fientes. De plus, il est très stable dans le milieu extérieur. Cette stabilité favorise sa transmission indirecte par l'intermédiaire de vecteurs passifs variés contaminés par les produits d'excrétion. Les animaux tels que les oiseaux sauvages, la souris, le rat, le chien et les insectes comme les moustiques peuvent être des vecteurs du virus et transmettre la maladie de Gumboro (Pagès-Mantè et al., 2004 ; Gilchrist, 2005;
Kasanga et al., 2008 ; Jeon et al., 2008 ; Park et al., 2010).

Au Sénégal, de nombreux facteurs sont supposés liés à l'apparition de la maladie de Gumboro dans les exploitations avicoles. Il existerait des facteurs qui, grâce à la résistance du virus aux différents traitements physiques et chimiques, favoriseraient la diffusion et la persistance du virus dans les exploitations.

L'objectif de cette étude est d'évaluer l'association entre des facteurs de risque supposés et l'apparition de la maladie dans les exploitations avicoles semi-intensives des régions de Dakar et Thiès en 2015. Pour cela, on explorera les liaisons de quatre facteurs à l'apparition de la maladie: la présence d'oiseaux domestiques ou sauvages à proximité des bâtiments d'élevage, la présence de rongeurs dans l'exploitation, la mauvaise gestion des animaux morts d'une quelconque affection et l'accès des personnes étrangères au niveau de l'exploitation.

\section{MATERIEL ET METHODES}

\section{Enquête sur les facteurs de risque supposés}

Une enquête épidémiologique a été conduite pour comparer, selon l'exposition à des facteurs de risque supposés, un groupe d'exploitations avicoles semi-intensives infectées par le virus de la maladie de Gumboro à un groupe d'exploitations avicoles semi-intensives indemnes de la maladie. L'enquête a été menée du $1^{\text {er }}$ décembre au 31 décembre 2015 dans les régions de Dakar et Thiès. Ces deux régions constituent avec les régions de Saint Louis et Louga, la zone des Niayes où le climat est marqué par l'influence 
de l'alizé maritime et l'existence d'une fraîcheur favorable aux activités avicoles (Figure 1). Les gestionnaires quotidiens des exploitations ont constitué les personnes cibles de cette étude. Des visites régulières ont été organisées afin d'interroger ces personnes sous forme d'un entretien. A cet effet, un seul questionnaire a été élaboré et portait sur la présence d'animaux domestiques ou sauvages à proximité des bâtiments d'élevage (dans un rayon de cinquante mètres), la présence de rongeurs dans le bâtiment d'élevage ou dans le magasin de stockage de l'aliment volaille, l'accès des personnes étrangères au niveau de l'exploitation et la mauvaise gestion des animaux morts d'une quelconque affection. La bonne gestion consiste à incinérer les sujets morts ou bien à les enfouir entre deux couches de chaux vive pour limiter la diffusion de l'agent pathogène.

\section{Echantillonnage}

Les exploitations avicoles semiintensives ont été choisies selon un mode aléatoire. En effet, 56 exploitations infectées et 112 exploitations indemnes ont été choisies au hasard à partir de lots d'exploitations infectées et indemnes suivies entre janvier et novembre 2015 dans le cadre d'une étude évaluant la prévalence de l'infection par le virus (Tableau 1). Les foyers de la maladie de Gumboro ont été suspectés sur la base de l'historique des épizooties, les manifestations cliniques et les lésions observées sur les organes des sujets malades, sacrifiés et autopsiés. Des prélèvements de bourses de Fabricius étaient effectués puis les tissus de bourses soumis à la technique RT-PCR pour détecter le virus en vue d'une confirmation de la suspicion clinique.

\section{Analyse statistique}

Le test du $\chi^{2}$ (khi-deux) a été utilisé pour comparer les proportions observées sur les échantillons indépendants selon la formule $\chi^{2}=\sum(\mathrm{ni}-\mathrm{fi})^{2} / \mathrm{fi}$ avec $\mathrm{ni}=$ fréquence d'observation, $\mathrm{fi}=$ fréquence théorique (Toma et al., 2010). Il a été fondé sur l'hypothèse nulle, à savoir l'absence d'une association statistique entre l'exposition à un facteur de risque supposé et l'apparition de la maladie. Ainsi, plus la valeur de $p$ est faible plus l'association observée est incompatible à l'hypothèse nulle et par conséquent plus le test est dit significatif. Une probabilité de $5 \%$ du rejet de cette hypothèse alors qu'elle est vraie a été retenue.

La force de l'association entre le facteur de risque supposé et l'apparition de la maladie a été quantifiée en calculant l'Odds Ratio, indicateur qui traduit l'intensité de cette relation. Ainsi, plus l'Odds Ratio est élevé, plus l'association entre le facteur de risque et la maladie est forte et si l'Odds Ratio calculé est égal à 1 , ceci correspond à l'absence d'association entre le facteur et la maladie.

Tableau 1 : Données sur les élevages avicoles concernés par l'enquête.

\begin{tabular}{lllc}
\hline & Effectifs & $\begin{array}{l}\text { Taille moyenne } \\
\text { des bandes }\end{array}$ & Production \\
\hline $\begin{array}{l}\text { Groupe des } \\
\text { élevages infectés }\end{array}$ & 56 & 256 & $96,34 \%$ élevages de poulets Chair \\
& & 289 & $4,66 \%$ élevages de poules pondeuses \\
\hline $\begin{array}{l}\text { Groupe des } \\
\text { élevages indemnes }\end{array}$ & 112 & $91,67 \%$ élevages de poulets Chair \\
\hline
\end{tabular}




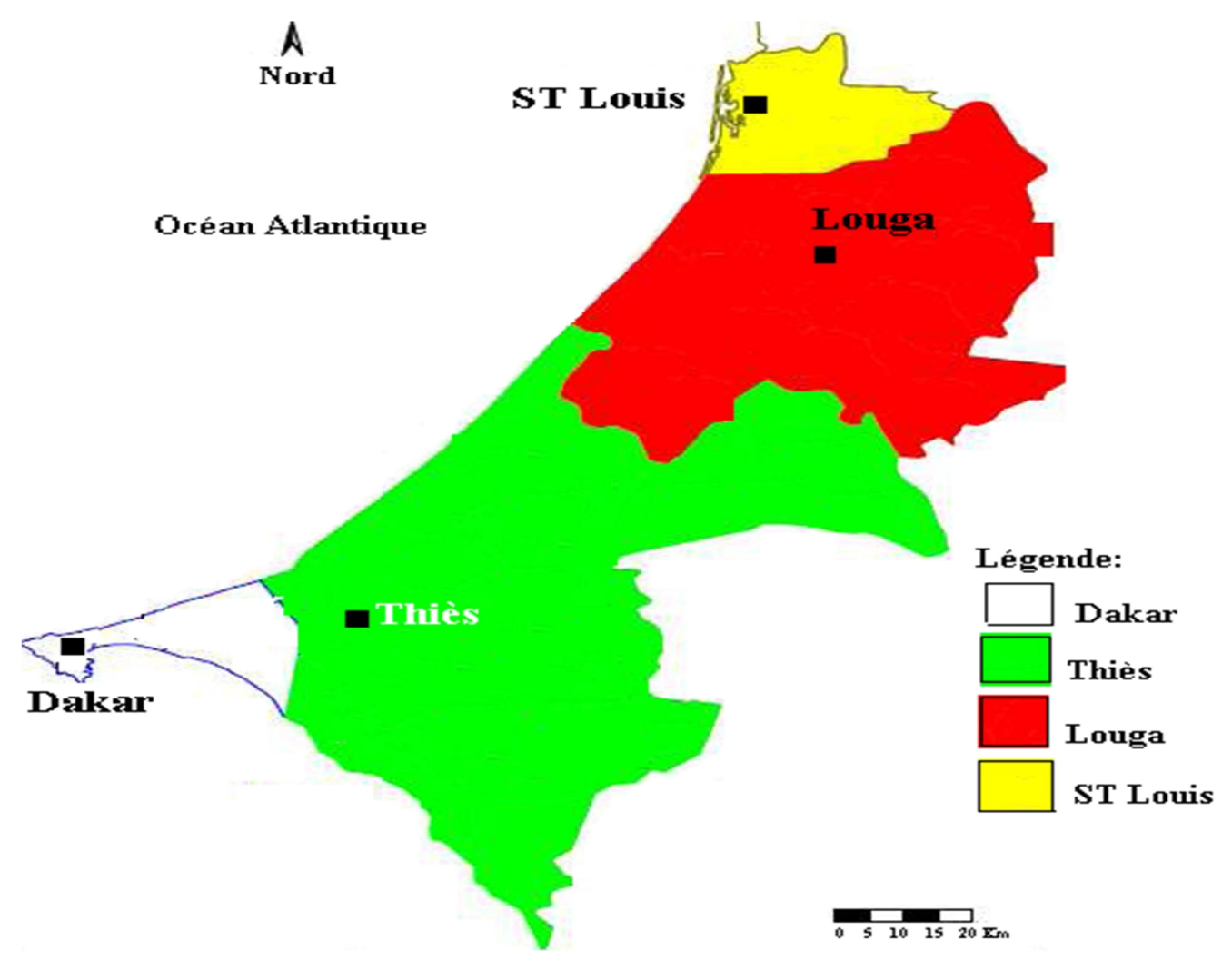

Figure 1 : Zone d'étude.

\section{RESULTATS}

Présence d'animaux domestiques ou sauvages à proximité des bâtiments d'élevage

Les résultats observés sur la présence d'animaux domestiques ou sauvages sont présentés dans le Tableau 2.

Les exploitations infectées par le virus de la maladie de Gumboro ont eu un pourcentage d'exposition de $25 \%$, légèrement supérieur à celui observé sur les exploitations indemnes $(18,75)$. L'écart est non significatif $\left(\chi^{2}=0,88 ; P=0,347\right)$ et l'indicateur qui traduit l'intensité de la relation, l'Odds Ratio est de 1,44 (IC $95 \%: 0,67 ; 3,12)$.

Présence de rongeurs dans le bâtiment d'élevage ou le magasin de stockage de l'aliment volaille

Les résultats observés sur la présence de rongeurs dans le bâtiment d'élevage ou le magasin de stockage de l'aliment sont présentés dans le Tableau 3.

Les exploitations infectées ont eu un pourcentage d'exposition de $83,92 \%$ très significativement supérieur à celui observé sur le lot des élevages indemnes $(18,75 \%)\left(\chi^{2}=\right.$ 65,83; $P<0,001)$. Les exploitations infectées étaient exposées dans un rapport de 22 fois (IC $95 \%$ : 9,61; 53,29) plus que les exploitations indemnes.

\section{Gestion des animaux morts d'une} quelconque maladie aviaire

Les résultats observés sur la gestion des animaux morts sont présentés dans le Tableau 4.

Les exploitations infectées et indemnes ont eu respectivement des pourcentages d'exposition de $60,71 \%$ et $16,96 \%$. Le pourcentage d'exposition est très significativement plus important sur le lot des exploitations infectées que sur le lot des 
exploitations indemnes $(\chi 2=33,09 ; P<$ $0,001)$. L'indicateur traduisant l'intensité de la relation, l'Odds Ratio est de 7,56 (IC 95\%: $3,65 ; 15,68)$.

\section{Accès des personnes étrangères au niveau} de l'exploitation

Les résultats observés sur l'accès des personnes étrangères au niveau de l'exploitation sont présentés dans le Tableau 5.

Les exploitations infectées et indemnes ont eu respectivement des pourcentages d'exposition de 69,64\% et $33,92 \%$. L'écart est très significatif $(\chi 2=$ $19,18 ; P<0,001)$ et l'Odds Ratio calculé est de 4,47 ( IC $\left._{95 \%}: 2,24 ; 8,92\right)$.

Tableau 2 : Animaux domestiques ou sauvages à proximité des bâtiments d'élevage.

\begin{tabular}{lccc}
\hline & $\begin{array}{c}\text { Exploitation } \\
\text { infectée }\end{array}$ & $\begin{array}{c}\text { Exploitation } \\
\text { Indemne }\end{array}$ & Total \\
\hline Présence & 14 & 21 & 35 \\
Absence & 42 & 91 & 133 \\
Total & 56 & 112 & 168 \\
Pourcentage d'exposition & 25 & 18,75 & \\
\hline
\end{tabular}

Tableau 3 : Présence de rongeurs dans le bâtiment d'élevage ou dans le magasin de stockage de l'aliment.

\begin{tabular}{lccc}
\hline & $\begin{array}{c}\text { Exploitation } \\
\text { infectée }\end{array}$ & $\begin{array}{c}\text { Exploitation } \\
\text { indemne }\end{array}$ & Total \\
\hline Présence & 47 & 21 & 68 \\
Absence & 09 & 91 & 100 \\
Total & 56 & 112 & 168 \\
Pourcentage d'exposition & 83,92 & 18,75 & \\
\hline
\end{tabular}

Tableau 4 : Gestion des animaux morts.

\begin{tabular}{lccc}
\hline & $\begin{array}{c}\text { Exploitation } \\
\text { infectée }\end{array}$ & $\begin{array}{c}\text { Exploitation } \\
\text { Indemne }\end{array}$ & Total \\
\hline Mauvaise gestion & 34 & 19 & 53 \\
Bonne gestion & 22 & 93 & 115 \\
Total & 56 & 112 & 168 \\
Pourcentage d'exposition & 60,71 & 16,96 & \\
\hline
\end{tabular}

Tableau 5 : Accès des personnes étrangères au niveau de l'exploitation.

\begin{tabular}{lccc}
\hline & $\begin{array}{c}\text { Exploitation } \\
\text { infectée }\end{array}$ & Exploitation indemne & Total \\
\hline Accès & 39 & 38 & 77 \\
Non accès & 17 & 74 & 91 \\
Total & 56 & 112 & 168 \\
Pourcentage d'exposition & 69,64 & 33,92 & \\
\hline
\end{tabular}




\section{DISCUSSION}

$\mathrm{Au}$ Sénégal, les épizooties de la maladie de Gumboro font leur apparition durant toute l'année et atteignent des pics au moment des grandes mises en place qui coïncident avec l'approche des grandes fêtes religieuses. La présence d'animaux domestiques ou sauvages dans les exploitations, l'accès des commerçants (collecteurs primaires et secondaires, détaillants, restaurateurs) au niveau des exploitations pendant ces périodes de déstockage massif sont souvent incriminés dans l'apparition de la maladie.

Si l'espèce Gallus gallus est reconnue comme étant la principale victime de la maladie, d'autres espèces d'animaux domestiques et sauvages peuvent être infectées par le virus, développer une forme inapparente de la maladie, jouer ainsi un rôle de réservoir et intervenir dans sa propagation.

Toutefois, le pourcentage d'exposition aux animaux domestiques ou sauvages des exploitations infectées est non significatif par rapport à celui observé sur le lot des exploitations indemnes $\left(\chi^{2}=0,88 ; P=\right.$ 0,347). De plus, l'Odds Ratio calculé est relativement très faible $(1,44)$.

Ce résultat obtenu indique une absence d'association entre ce facteur de risque et l'apparition de la maladie. Les animaux domestiques sont aussi bien élevés par des aviculteurs occasionnels que par les aviculteurs expérimentés (Etienne, 2002). Toutefois, il a été observé, dans la plupart des exploitations visitées, de petits effectifs de poulets traditionnels élevés dans des poulaillers construits à des distances de plus d'une cinquantaine de mètres par rapport aux bâtiments d'élevage. Les canards et les pigeons sont rarement élevés. Les chiens errants, les corbeaux et les vautours sont fréquemment observés aux alentours des exploitations à la recherche de cadavres de poulets. Ils pourraient s'infecter de manière inapparente en consommant les sujets morts et constituer une source d'infection moins intense, insidieuse et durable où se contaminent des vecteurs compétents. Ces derniers assureraient le transport du virus vers les bâtiments d'élevage.
L'absence d'une liaison significative entre ce facteur de risque et l'apparition de la maladie ne signifie pas absolument que les animaux domestiques et sauvages ne jouent aucun rôle dans la transmission du virus. Ils joueraient un rôle secondaire par rapport à d'autres facteurs de risque. En effet, une étude menée en Coré par Jeon et al. (2008), a rapporté l'infection des oiseaux sauvages par la souche hypervirulente du virus de la maladie de Gumboro. Par ailleurs, une étude conduite par Pages-mante et al. (2004), évaluant la possibilité que le chien soit un éventuel porteur du virus après avoir mangé volontairement ou accidentellement des poulets infectés, a démontré l'excrétion de celui-ci sous sa forme virulente dans les fèces. Les chiens errants, en nombre important dans les endroits visités pourraient jouer un rôle important dans la diffusion du virus, en tant que réservoir.

Le pourcentage d'exposition à la présence de rongeurs observé sur le lot des exploitations infectées est significativement supérieur à celui observé sur le lot des exploitations indemnes $\left(\chi^{2}=65,83 ; P<\right.$ 0,001). La présence de rongeurs a été fréquemment évoquée par les gestionnaires des élevages. En fait, la vétusté des bâtiments d'élevage semi-ouverts et des magasins de stockage de l'aliment favorise la colonisation de ces locaux par les rats et les souris. Ils sont difficilement combattus par les aviculteurs qui utilisent diverses stratégies et moyens de lutte. Le résultat obtenu pourrait expliquer une implication de ces rongeurs dans la transmission $\mathrm{du}$ virus au sein d'une exploitation et même entre des exploitations voisines. Ils se contamineraient à partir des fientes pour propager le virus. Ceci serait facilité par le stockage du fumier en plein air, à côté des bâtiments d'élevage. Cette pratique a été rapportée au Bénin par Boko et al. (2015).

Une étude menée par Park et al. (2010) a confirmé la viabilité et la pathogénicité du virus une fois excrété dans les fèces par la souris. Par conséquent, ces rongeurs pourraient jouer un rôle important dans la contamination de l'aliment et de l'eau distribués aux poulets. 
La destruction des animaux morts d'une quelconque maladie aviaire s'impose, dans des conditions permettant d'éliminer l'agent pathogène responsable. Toutefois, les résultats obtenus révèlent que $60,71 \%$ des exploitations infectées par le virus étaient exposées auparavant à une mauvaise gestion des sujets malades d'autres affections aviaires. Ce pourcentage observé est significativement plus important $(\chi 2=33,09 ; P<0,001)$ que celui observé sur le lot des exploitations indemnes. En effet, dans la majeure partie des exploitations infectées, il a été observé que les aviculteurs ne réagissaient pas spontanément aux premiers signes cliniques d'une quelconque affection. Les premiers cas sont souvent laissés dans le bâtiment d'élevage jusqu'à ce qu'ils soient morts. Les cadavres ne sont ni incinérés, ni enfouis entre deux couches de chaux vive. Ils les entasser en plein air dans un endroit de l'exploitation qu'ils érigent en dépotoir. Les cadavres doivent être détruits par incinération ou enfouissement pour que le virus soit effectivement détruit car, plusieurs études rapportées ont évoqué la forte résistance du virus dans le milieu extérieur (Lukert et Saif, 2003).

La maladie de Gumboro peut être transmise d'une exploitation à l'autre par l'homme, en tant que simple support d'un transport mécanique, sans multiplication du virus. L'homme mérite une attention particulière à cause de ses nombreux déplacements et de ses activités diverses qui augmentent son rôle épidémiologique de potentiel «transporteur». L'homme peut véhiculer le virus sur son corps, ses habits et ses chaussures d'une exploitation infectée vers une exploitation saine. Les exploitations infectées ont un pourcentage d'exposition aux déplacements et activités des commerçants (collecteurs primaires, secondaires, détaillants et restaurateurs) et des aviculteurs significativement supérieur à celui observé sur le lot des exploitations indemnes $(\chi 2=19,18$; $P<0,001)$. En effet, un accès facile est surtout observé au niveau des petites exploitations qui sont très proches des axes routiers et des villes. Plus nombreuses, elles sont caractérisées par des effectifs de bandes qui varient entre 50 et 400 sujets (Diagne, 2008). Peu expérimentés, les éleveurs de ces exploitations disposent des moyens financiers très limités les exposant durant toute l'année à la maladie de Gumboro.

\section{Conclusion}

Les résultats obtenus dans cette étude indiquent que la présence de rongeurs, la mauvaise gestion des cadavres et l'accès des personnes étrangères au niveau des exploitations avicoles sont autant de facteurs fortement liés à l'apparition de la maladie de Gumboro. Ces facteurs s'associent pour favoriser la diffusion du virus et l'apparition de la maladie. Les chiens errants et les oiseaux rapaces, en consommant les cadavres déposés en plein air par une bonne partie des aviculteurs rencontrés, disséminent le virus dans un rayon important. Les rats et les souris se contaminent au niveau des dépotoirs et transmettent le virus aux poulets par l'intermédiaire de l'aliment et de l'eau distribués. Compte tenu des difficultés de détruire le virus dans les bâtiments d'élevage par le nettoyage et la désinfection, une lutte axée sur la sensibilisation des aviculteurs sur la conduite à tenir vis-à-vis des sujets malades et des cadavres contribuerait à réduire la diffusion $\mathrm{du}$ virus dans les exploitations avicoles semi-intensives au Sénégal.

\section{CONFLIT D'INTERETS}

Les auteurs déclarent qu'il n'existe aucun conflit d'intérêts concernant la publication de cet article.

\section{CONTRIBUTIONS DES AUTEURS}

La thématique de recherche a été mise en forme par AB, MML et RBA ont suivi et supervisé le travail de recherche. Ils se sont aussi impliqués dans la correction et la mise en forme du manuscrit.

\section{REMERCIEMENTS}

Les auteurs remercient le personnel de la section virologie et de la section pathologies aviaires du LNERV de l'Institut Sénégalais de Recherches Agronomiques (ISRA) de Dakar, les Aviculteurs de Dakar et Thiès, les Docteurs vétérinaires du privé et du 
public, les Ingénieurs des travaux d'élevage, les PER de l'ISFAR/UT, M. Ousmane KANE Enseignant-Chercheur au DPA/ISFAR/UT et les Agents Techniques d'Elevage, d'avoir considérablement participé dans la réalisation de cette étude.

\section{REFERENCES}

Boko MA, Dougnon TV, Bankolé HS, Dougnon TJ, Ahouangninou C, Cledjo P, Soumanou M. 2015. Pratiques d'élevage avicole au Sud-Bénin (Afrique de l'Ouest) et impacts sur l'hygiène des fumiers produits. International Journal of Biological and Chemical Sciences, 9(6): $\quad$ 2740-2753. DOI: http://dx.doi.org/ 10.4314/ijbcs.v9i6.18.

Badji A, Diallo AA, Ducatez M, Tall F, Diop M, Mbengue M, Lo MM, Thiongane $\mathrm{Y}$, Guerin JL, Alambedji RB. 2016. Genetic evolution of infectious bursal disease virus in Senegal, 1999-2014. Journal of veterinary Medicine and Animal Health, 8(12): 237-243. DOI: 10.5897/ JVMAH2016.0521

Diagne MM. 2008. Analyse de la compétitivité de la filière avicole semiindustrielle dans la zone des Niayes. Mémoire de fin d'études ENSA, Thiès (Sénégal), 60p.

Etienne F. 2002. Stratégies de prévention de la maladie de Gumboro dans les élevages semi-industriels de la région de Dakar, Sénégal. Thèse: Médecine Vétérinaire, Toulouse, TOU 3: 4018.

Gilchrist P. 2005. Involvement of free-flying wild birds in the spread of the viruses of avian influenza, Newcastle disease and infectious bursal disease from Poultry products to commercial Poultry. World's Poultry Science Journal, 61(02): 198214.

http://dx.doi.org/10.1079/WPS200451.

Jeon WJ, Lee EK, Joh SJ, Kwon JH, Yang CB, Yoon YS, Choi KS. 2008. Very virulent infectious bursal disease virus isolated from wild birds in Korea: epidemiological implications. Virus
Research, 137(1): 153-156. DOI: 10.1016/j.virusres.

Kasanga CJ, Yamaguchi T, Wambura PN, Munang'andu HM, Ohya K, Fukushi H. 2008. Detection of infectious bursal disease virus (IBDV) genome in freeliving pigeon and guinea fowl in Africa suggests involvement of wild birds in the epidemiology of IBDV. Virus Genes, 36: 521-529. DOI: 10.1007/s11262-008-0219-z.

Lukert PD, Saif YM. 2003. Infectious bursal disease. In Diseases of Poultry $\left(11^{\text {th }}\right.$ edn), Saif YM, Barnes HJ, Fadly AM, Glisson JR, Mcdougald LR, Swayne DE (eds). Iowa State University Press: Ames, USA; 161-179.

Muller H, Islam MR, Rauea R. 2003. Research on infectious bursal disease-the past, the present and the future. Veterinary Microbiology, 97: 153-165.

Pages-mante A, Torrents D, Maldonado J, Saubi N. 2004. Dogs as potential carriers of infectious bursal disease virus. Journal of Avian Pathology, 33(2): 205209. DOI: 10.1080/03079450420001 95821.

Park MJ, Park JH, Kwon HM. 2010. Mice as potential carriers of infectious bursal disease virus in chickens. The Veterinary Journal, 183: 352-354. DOI: 10.1016/j.tvj1.2008.12.005.

Rabeson FA. 2010. Enquête sérologique sur la maladie de Gumboro et biomoléculaire sur l'Influenza aviaire hautement pathogène en aviculture traditionnelle au Sénégal. Mémoire de Diplôme de Master II en Santé Publique Vétérinaire, Dakar (Sénégal), 41p.

Sharma JM, Kim IJ, Rautenschlein S, Yeh HY. 2000. Infectious bursal disease virus of chickens: pathogenesis and immunosuppression. Developmental \& Comparative Immunology, 4: 223-235.

Toma B, Dufour B, Bénet JJ, Sanaa M, Shaw A, Moutou F. 2010. Epidémiologie appliquée à la lutte collective contre les maladies animales transmissibles majeures. AEEMA, $600 \mathrm{p}$. 